\title{
Synthesis and nonlinear optical properties of some donor-acceptor oxadiazoles
}

\author{
Sabir H. Mashraqui ${ }^{\text {a,* }}$, Rajesh S. Kenny ${ }^{\text {a }}$, Shailesh G. Ghadigaonkar ${ }^{\text {a }}$, Anu Krishnan ${ }^{\text {b }}$, \\ Mily Bhattacharya ${ }^{\mathrm{b}}$, Puspendu K. Das ${ }^{\mathrm{b}}$ \\ ${ }^{a}$ Department of Chemistry, University of Mumbai, Vidyanagari, Santacruz (E), Mumbai 400098, India \\ ${ }^{\mathrm{b}}$ Department of Inorganic and Physical Chemistry, Indian Institute of Science, Bangalore 560012, India
}

\begin{abstract}
Heterocyclic 1,3,4-oxadiazole moiety has been used as a new $\pi$-conjugation relay to prepare a number of donor-acceptor molecules carrying a $\pi$-electron-rich aromatic ring as a donor and either a $p$-nitrophenyl or a pyridine/pyridinium ring as an acceptor. Several of these oxadiazoles display significant second-order molecular nonlinearity, $\beta\left(14-124 \times 10^{-30}\right.$ esu $)$ and provide the basis for future design of efficient nonlinear optical materials having the oxadiazole core.
\end{abstract}

PACS: 42.70.J; 78.66.Q; 42.65.K

Keywords: Nonlinear optics; First hyperpolarizability; Donor-acceptor oxadiazoles; Synthesis; UV-visible; Hyper-Rayleigh scattering technique; Thermal decomposition

\section{Introduction}

Organic molecules and materials possessing large second-order nonlinearity are sought for potential applications in optical communication, information storage, optical switching, etc. [1-3]. The design strategy, used by many with success involves connecting donor (D) and acceptor (A) groups at the terminal positions of a $\pi$ bridge to create highly polarized molecules which could exhibit large molecular nonlinearity. To date, the types of $\pi$-bridges investigated for developing efficient NLO materials and molecules include DA olefines [4,5], acetylenes [6], azo bridges [7], aromatic [8] and heteroaromatic rings [9,10]. Although, push-pull polyenes generally show very large first hyperpolarizability $\beta$, their thermal stability is not satisfactory. On

\footnotetext{
*Corresponding author. Tel.: +91-2226526119; fax: +912226528457.

E-mail address: sh_mashraqui@yahoo.com (S.H. Mashraqui).
}

the other hand, aromatic D-A molecules are more stable but they exhibit relatively lower $\beta$ values. Incorporation of benzene rings into the aliphatic push-pull polyenes is found to saturate molecular nonlinearity [11]. To overcome this problem, several groups [9-15] have developed NLO chromophores containing easily polarizable fivemembered heteroaromatic rings. Heterorings such as furan and thiophene, due to their relatively lower aromatic stabilization energy than benzene are reported to provide more effective $\pi$-conjugation between $\mathrm{D}$ and $\mathrm{A}$, resulting in larger nonlinearities [11]. However, recent semiempirical calculations [16] have discounted the relevance of aromatic delocalization energies in the magnitude of NLO response of heterocyclic compounds but stressed on the need of tuning electronic factor to achieve large nonlinearity.

In the search of new NLO motifs among heterocyclic compounds, here we have used the 1,3,4-oxadiazole ring as a $\pi$-bridge on the ground that its reduced aromaticity may offer better prospects for $\pi$-electron delocalization across the D-A links. Substitutions at the 2 and 5 positions of the oxadiazole core have been made to ensure that the oxadiazole core, indeed, acts as a 
$\pi$-conjugated backbone. Various aromatic donors and acceptors have been used to tune the electronic factor and understand the origin of the second order nonlinearity in these molecules.

\section{Synthesis of donor-acceptor oxadiazoles}

The first series of D-A oxadiazoles synthesized by us consists of various $\pi$-rich aromatic heterocycles as donors and the $p$-nitrophenyl moiety as the acceptor to generate structures 5a-f (Fig. 1). The known 2-chloromethyl oxadiazole $\mathbf{2}$ was prepared by heating hydrazide 1 with chloroacetyl chloride in dioxane solvent. The reaction of $\mathbf{2}$ with an equimolar amount of $\mathrm{PPh}_{3}$ readily produced phosphonium salt 3 . The latter was subjected to the Wittig olefination with aldehydes 4a-f using a general procedure involving treatment with anhyd. $\mathrm{K}_{2} \mathrm{CO}_{3}$ in methanol at room temperature. The work-up of the reaction gave crude products showing a major single spot on the TLC. Purification was carried out on a silica column to obtain the oxadiazoles $\mathbf{5 a}-\mathbf{f}$ in $55-75 \%$ yields [17]. These compounds, isolated as the major isomers were assigned $(E)$-geometry on the basis of their $500 \mathrm{MHz}{ }^{1} \mathrm{H}$ NMR spectra, which revealed olefinic coupling constants in between 18 and $21 \mathrm{~Hz}$.

Another series of D-A oxadiazole-A, 8a-f was synthesized by replacing the $p$-nitrophenyl moiety present in 5a-f with the pyridine unit as the acceptor. The synthesis of 8a-f begins with pyridyl-oxadiazole 7 [18], which was prepared in an improved yield (dioxan/ microwave heating) by reacting hydrazide 6 with acetyl chloride. Base catalyzed condensation of 7 with aldehydes $\mathbf{4 a}-\mathbf{f}$ led to the formation of pyridyl oxadiazoles 8a-f [19] with $(E)$ stereochemistry as deduced from the $500 \mathrm{MHz}{ }^{1} \mathrm{H}$ NMR spectra. Next, in order to enhance the acceptor strength, compounds 8a-f were treated with $\mathrm{CH}_{3} \mathrm{I}$ in acetonitrile solvent to obtain high yields of the corresponding $N$-methyl pyridinium salts $9 \mathbf{a}-\mathbf{f}$ (Fig. 1).

The UV-vis absorption data and the first hyperpolarizability, $\beta$ of the oxadiazole compounds were measured by the hyper-Rayleigh scattering (HRS) technique $[20,21]$ in solution at $1064 \mathrm{~nm}$ using the external reference method [22] and are reported in Table 1. The molecular hyperpolarizability was not corrected for two photon fluorescence. The static hyperpolarizability $\left(\beta_{0}\right)$ was calculated using the two state model using the expression [23]
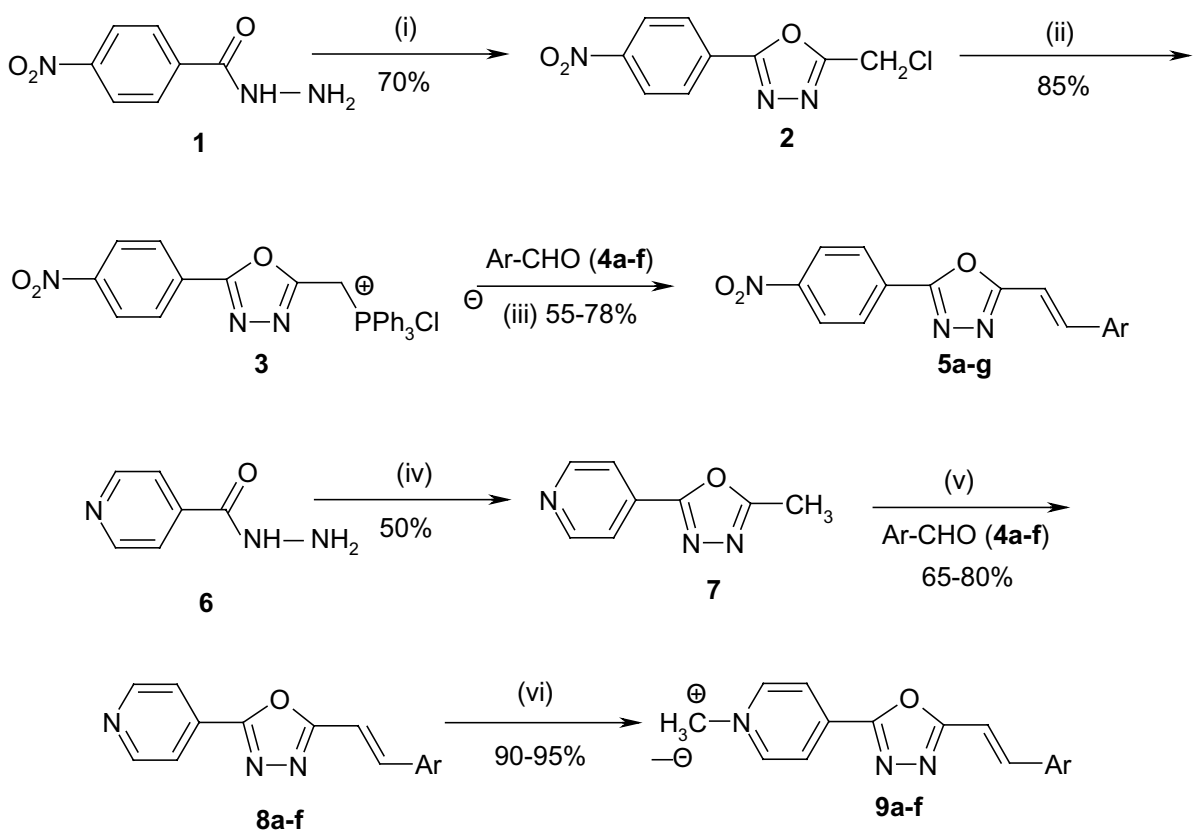

$4 a ; 5 a ; 8 a ; 9 a ; A r=P h-$ $4 \mathrm{~b} ; 5 \mathrm{~b} ; 8 \mathrm{~b} ; 9 \mathrm{~b} ; \mathrm{Ar}=4-\mathrm{MeO}-\mathrm{Ph}-$ $4 c ; 5 c ; 8 c ; 9 c ; A r=4-\mathrm{Me}_{2} \mathrm{~N}-\mathrm{Ph}-$ $4 d ; 5 d ; 8 d ; 9 d ; A r=2$-furyl$4 e ; 5 e ; 8 e ; 9 e ; A r=2$-thienyl4f; 5f; 8f; 9f; Ar = 1-Methyl 2-pyrrolyl-

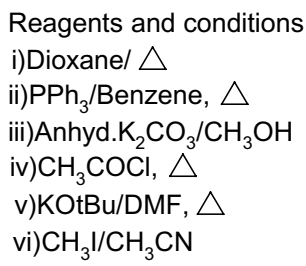

Reagents and conditions i)Dioxane/ $\triangle$ ii) $\mathrm{PPh}_{3} /$ Benzene, $\triangle$ iii)Anhyd. $\mathrm{K}_{2} \mathrm{CO}_{3} / \mathrm{CH}_{3} \mathrm{OH}$ iv) $\mathrm{CH}_{3} \mathrm{COCl}, \triangle$ v)KOtBu/DMF, $\triangle$ vi) $\mathrm{CH}_{3} \mathrm{l} / \mathrm{CH}_{3} \mathrm{CN}$

Fig. 1. Synthetic sequences for D-A oxadiazoles 5a-g, 8a-f and 9a-f. 
Table 1

Electronic absorption and hyperpolarizability of D-A oxadiazoles $5 \mathbf{a}-\mathbf{f}, \mathbf{8 a}-\mathbf{f}$ and $9 \mathbf{a}-\mathbf{f}$

\begin{tabular}{|c|c|c|c|c|c|c|c|c|c|c|c|c|c|c|}
\hline Comp. & $\begin{array}{l}\lambda_{\max } \\
\mathrm{nm}\end{array}$ & $\begin{array}{l}\beta \times 10^{-30 \mathrm{a}} \\
\text { esu }\end{array}$ & $\begin{array}{l}\beta_{0} \times 10^{-30} \\
\text { esu }\end{array}$ & $\begin{array}{l}T_{\mathrm{d}} \\
{ }^{\circ} \mathrm{C}\end{array}$ & Comp. & $\begin{array}{l}\lambda_{\max } \\
\mathrm{nm}\end{array}$ & $\begin{array}{l}\beta \times 10^{-30} \\
\text { esu }\end{array}$ & $\begin{array}{l}\beta_{0} \times 10^{-30} \\
\text { esu }\end{array}$ & $\begin{array}{l}T_{\mathrm{d}} \\
{ }^{\circ} \mathrm{C}\end{array}$ & Comp. & $\begin{array}{l}\lambda_{\max } \\
\mathrm{nm}\end{array}$ & $\begin{array}{l}\beta \times 10^{-30} \\
\text { esu }\end{array}$ & $\begin{array}{l}\beta_{0} \times 10^{-30} \\
\text { esu }\end{array}$ & $T_{\mathrm{d}}{ }^{\circ} \mathrm{C}$ \\
\hline $5 a$ & 338 & 34.0 & 18.3 & 248 & $8 a$ & 317 & 46.0 & 27.2 & 212 & $9 a$ & 353 & 77.0 & 38.5 & 248 \\
\hline $5 b$ & 360 & 49.0 & 23.5 & 204 & $8 b$ & 340 & 50.0 & 28.0 & - & $9 b$ & 382 & 94.7 & 39.5 & 206 \\
\hline $5 c$ & 420 & 60.1 & 19.1 & 203 & $8 c$ & 400 & 60.0 & 23.0 & 279 & $9 c$ & 459 & 123.6 & 25.7 & 246 \\
\hline $5 d$ & 357 & 39.7 & 19.5 & 223 & $8 d$ & 341 & 14.1 & 7.8 & 199 & 9d & 377 & 59.6 & 26.0 & $>410$ \\
\hline $5 e$ & 358 & 32.6 & 15.8 & - & $8 e$ & 343 & 28.1 & 14.8 & 223 & $9 e$ & 374 & 57.3 & 26.0 & 200 \\
\hline $5 f$ & 390 & 38.1 & 15.2 & 177 & $8 f$ & 374 & 28.0 & 12.7 & 254 & 9f & 430 & - & - & 226 \\
\hline
\end{tabular}

${ }^{\mathrm{a}}$ In the external reference method the measured $\beta$ values were calibrated against paranitroaniline in chloroform and in some cases methanol as the standard. The $\beta$ values used for paranitroaniline are $17.8 \times 10^{-30}$ esu and $22.0 \times 10^{-30}$ esu in chloroform and methanol, respectively.

$\beta / \beta_{0}=\left(\omega_{0}\right)^{4} /\left[\left(\omega_{0}^{2}-\omega^{2}\right)\left(\omega_{0}^{2}-4 \omega^{2}\right)\right]$

where $\omega_{0}$ is the single photon absorption maximum of the molecule in wavenumbers, and $\omega$ is the laser fundamental. The dispersion free hyperpolarizability data calculated using Eq. (1) are also listed in Table 1.

\section{Results and discussion}

The absorption band for phenyl oxadiazole 5a appears at $338 \mathrm{~nm}$, which is shifted to the red by $82 \mathrm{~nm}$ for the highly polarized oxadiazole $5 \mathbf{c}\left(\lambda_{\max }\right.$ at $\left.420 \mathrm{~nm}\right)$ carrying a strong donor group, dimethylaniline at the para position of the aryl ring. This is accompanied by a steady increase in $\beta$ from $\mathbf{5 a}$ to $\mathbf{5 c}$ which is expected along with the red shift in absorption band because of dispersion characteristics of the second-order nonlinearity. For the other oxadiazoles $\mathbf{5 d}$, $5 \mathbf{e}$ and $\mathbf{5 f}$, having $\pi$ donor chromophores of intermediate strength, the charge transfer (CT) band is located within the 338-420 $\mathrm{nm}$ range. Among 5a-f, in accord with the relative donor strength, the lowest $\beta_{0}$ is observed for the phenyl oxadiazoles 5e and 5 f $\left(\sim 15.0 \times 10^{-30}\right.$ esu $)$ whereas oxadiazoles $\mathbf{5 b}$ and $\mathbf{5 c}$ carrying strong donors, exhibit the $\beta_{0}$ value of $\sim 22 \times 10^{-30}$ esu. Surprisingly, in oxadiazoles 5d-f, the presence of aromatic heterocyclic donors with lower resonance stabilization energy compared to benzene, such as thiophene, furan or pyrrole is not conducive for enhancing the dispersion free hyperpolarizability. We, thus, infer that in oxadiazoles conventional donors such as $-\mathrm{NMe}_{2}$ or $-\mathrm{OMe}$ are preferred over others for enhancing the second order nonlinearity.

The CT bands for oxadiazoles 8a-f appear in the range between 317 and $400 \mathrm{~nm}$, which are shifted to the red by 36 to $59 \mathrm{~nm}$ in the corresponding ionic compounds 9a-f $\left(\lambda_{\max }=353-460 \mathrm{~nm}\right)$, an observation which is consistent with the increased $\pi$-acceptor ability of the pyridinium ring compared to the pyridine ring. The red shift in absorption is also accompanied with an upward shift in the $\beta$ values. The first static hyperpolarizability $\beta_{0}$ in the neutral oxadiazoles, 8a-f was found to vary from a low of $8 \times 10^{-30}$ esu for the furan analog, $8 \mathbf{d}$ to a high of $28 \times 10^{-30}$ esu for oxadiazole $\mathbf{8 b}$, carrying the methoxy donor. In concurrence with the series $\mathbf{5}$ and contrary to our own expectation, all oxadiazoles $\mathbf{8 d}-\mathbf{f}$, bearing the heterorings furan, thiophene and pyrrole display lower $\beta_{0}$ values than the simple phenyl oxadiazole $8 \mathbf{a}$. The better $\pi$-acceptor property of the pyridinium ring is clearly reflected by higher $\beta_{0}$ values for the ionic 9a-f relative to $\mathbf{8 a}-\mathbf{f}$. For the furan donor, 9d, the increase in $\beta_{0}\left(23 \times 10^{-30} \mathrm{esu}\right)$ is almost three times of that of the neutral $\mathbf{8 d}\left(7.8 \times 10^{-30}\right.$ esu). All the compounds 9a-f compare well with the known NLO prototype, 4-dimethylamino-4'-nitrostilbene $(\beta=55.2 \times$ $10^{-30}$ esu in chloroform).

The thermal stability of the compounds was checked by scanning calorimetry and the thermal decomposition temperatures $\left(T_{\mathrm{d}}\right)$ are listed in Table 1 . The compounds are all quite stable and $T_{\mathrm{d}} \mathrm{S}$ are higher than $200{ }^{\circ} \mathrm{C}$. The $T_{\mathrm{d}}$ of compound $9 \mathbf{d}$ is not exactly determinable since it decomposes with melting at a temperature higher than $410{ }^{\circ} \mathrm{C}$. Compound $9 \mathrm{~b}$ which has the highest hyperpolarizability among the heterocyclic donor acceptor compounds studied here has a decomposition temperature of $248{ }^{\circ} \mathrm{C}$. The thermal stability of this class of compounds is comparable and in some cases lower than that of DANS $\left(T_{\mathrm{d}}=290{ }^{\circ} \mathrm{C}\right)[7]$.

\section{Conclusion}

In conclusion, it is clear from this study that certain D-A oxadiazoles, particularly those containing the $p$-methoxyphenyl $/ p$-dimethylaminophenyl donor and the pyridinium acceptor, show large second-order 
nonlinearity. The oxadiazole core can be used as an effective $\pi$-bridge in the design of new organic NLO molecules. However, it appears that heterocycles having lower aromatic stabilization energy than benzene, such as furan, thiophene and pyrrole, do not contribute significantly to the hyperpolarizability which is contrary to what is known in the context of second order NLO materials. Further variation in the type of D/A groups and the $\pi$-conjugation network containing the oxadiazole ring in the core structure are necessary for reaching a concrete inference.

\section{Acknowledgements}

SHM and PKD thank C.S.I.R., New Delhi for supporting this research. We also thank S. Ramakrishnan for letting us use his differential scanning calorimeter.

\section{References}

[1] D.S. Chemla, J. Zyss, in: Nonlinear Optical Properties of Organic Molecules and Crystals, vols. 1 and 2, Academic Press, New York, 1987.

[2] P.N. Prasad, D.R. Ulrich, Nonlinear Optical and Electroactive Polymers, Plenum, New York, 1988.

[3] D.R. Kanis, M. Ratner, T.J. Marks, Chem. Rev. 94 (1994) 193.

[4] S.R. Marder, L.-T. Cheng, B.G. Tiemann, A.C. Friedli, M. Blanchard-Desce, J.W. Perry, J. Skindhoj, Science 263 (1994) 511.

[5] M. Blanchard-Desce, C. Runser, A. Fort, M. Barzoukas, J.-M. Lehn, V. Ploy, V. Alain, Chem. Phys. 199 (1995) 253.

[6] L.-T. Cheng, W. Tam, S.H. Stevenson, G.R. Meredith, G. Rikken, S.R. Marder, J. Phys. Chem. 95 (1991) 10631.

[7] C.R. Moylan, R.J. Twieg, V.Y. Lee, S.A. Swanson, K.M. Betterton, R.D. Miller, J. Am. Chem. Soc. 115 (1993) 12599.

[8] L.-T. Cheng, W. Tam, S.R. Marder, A.E. Stiegman, G. Rikken, C.W. Spangler, J. Phys. Chem. 95 (1991) 10643.

[9] V.P. Rao, Y.M. Cai, A.K.Y. Jen, Chem. Comm. (1994) 1689.

[10] V.P. Rao, A.K.Y. Jen, K.Y. Wong, K.J. Drost, Chem. Comm. (1993) 1118.
[11] P.R. Varanasi, A.K.Y. Jen, J. Chandrasekhar, I.N.N. Namboothiri, A. Rathna, J. Am. Chem. Soc. 118 (1996) 12443.

[12] O. Kim, J.-M. Lehn, Chem. Phys. Lett. 255 (1996) 147.

[13] L.R. Dalton, A.W. Harper, B.H. Robinson, Proc. Natl. Acad. Sci. USA 94 (1997) 4842.

[14] T.T. Nguyen, M. Salle, J. Delaunay, A. Riou, P. Richomme, J.M. Raimundo, A. Gorgues, I. Ledoux, C. Dhenaut, J. Zyss, J. Orduna, J. Garin, J. Mater. Chem. 8 (1998) 1185.

[15] C.-F. Shu, Y.-K. Wang, J. Mater. Chem. 8 (1998) 833.

[16] E.M. Breitung, C.-F. Shu, R.J. McMahon, J. Am. Chem. Soc. 122 (2000) 1154.

[17] A representative procedure for the preparation of compounds 5af: To a solution of phosphonium salt $3(2.5 \mathrm{mmol})$ and freshly distilled benzaldehyde $(3.0 \mathrm{mmol})$ in $50 \mathrm{ml}$ of dry methanol was added anhyd. $\mathrm{K}_{2} \mathrm{CO}_{3}(10 \mathrm{mmol})$ and the reaction mixture was stirred at room temperature for $24 \mathrm{~h}$. The reaction mixture was then diluted with water and precipitated solid filtered and air dried. The crude product showing a major single spot on TLC was purified by $\mathrm{SiO}_{2}$ column (elution with $\mathrm{CHCl}_{3}$ : hexane, 1:1) to give 5a as solid in $75 \%$ yield. Crystallisation from ethyl alcohol afforded an analytical sample, m.p. $188-190{ }^{\circ} \mathrm{C}$; Anal. Calcd for $\mathrm{C}_{16} \mathrm{H}_{11} \mathrm{~N}_{3} \mathrm{O}_{3}$ : C, 65.51; H, 3.78; N, 14.36. Found: C, 65.34; $\mathrm{H}$, 3.44; N, 14.56; ${ }^{1} \mathrm{H}$ NMR $\left(500 \mathrm{MHz} ; \mathrm{CDCl}_{3}\right) \delta 7.1(1 \mathrm{H}, \mathrm{d}, J=16$ $\mathrm{Hz}), 7.65(1 \mathrm{H}, \mathrm{d}, J=16 \mathrm{~Hz}), 7.4-7.5(5 \mathrm{H}, \mathrm{m}), 8.25(2 \mathrm{H}, \mathrm{d}, J=8$ $\mathrm{Hz}), 8.35(2 \mathrm{H}, \mathrm{d}, J=8 \mathrm{~Hz})$.

[18] V.L. Pachhamia, A.R. Parekh, J. Ind. Chem. Soc. 66 (1989) 250.

[19] A representative procedure for the preparation of compounds 8af: To a solution of oxadiazole $7(5 \mathrm{mmol})$-anisaldehyde $(7.5$ mmol) in dry DMF $(15 \mathrm{ml})$ was added a catalytic amount of potassium $t$-butoxide $(1 \mathrm{mmol})$. The reaction was stirred under $\mathrm{N}_{2}$ atm for $5 \mathrm{~h}$ and then decomposed by pouring into cold water. The precipitated solid was filtered, washed with water and dried. Repeated crystallization from ethyl alcohol afforded $\mathbf{8 b}$ as white solid, m.p. $165-68{ }^{\circ} \mathrm{C}$ in $45 \%$ yield. Anal. Calcd for $\mathrm{C}_{16} \mathrm{H}_{13} \mathrm{~N}_{3} \mathrm{O}_{2}$ : 68.81; H, 4.69; N, 15.04. Found: C, 68.55; H, 4.40; N, 15.42; ${ }^{1} \mathrm{H}$ NMR $\left(500 \mathrm{MHz} ; \mathrm{CDCl}_{3}\right) \delta 3.86(3 \mathrm{H}, \mathrm{S}), 7.0(1 \mathrm{H}, \mathrm{d}, J=21 \mathrm{~Hz})$, $7.2(2 \mathrm{H}, \mathrm{d}, J=14 \mathrm{~Hz}), 7.67(1 \mathrm{H}, \mathrm{d}, J=21 \mathrm{~Hz}), 8.2(2 \mathrm{H}, \mathrm{d}, J=14$ $\mathrm{Hz}), 8.7(2 \mathrm{H}, \mathrm{d}, J=7 \mathrm{~Hz}), 8.8(2 \mathrm{H}, \mathrm{d}, J=7 \mathrm{~Hz})$.

[20] K. Clays, A. Persoons, Phy. Rev. Lett. 66 (1991) 2980.

[21] A. Krishnan, S.K. Pal, P. Nandakumar, A.G. Samuelson, P.K. Das, Chem. Phys. 265 (2001) 313.

[22] T. Kodaira, A. Watanabe, O. Ito, M. Matsuda, K. Clays, A. Persoons, J. Chem. Soc., Faraday Trans. 93 (1997) 3039.

[23] B.J. Orr, J. Ward, Mol. Phys. 20 (1971) 513. 